PROCEEDINGS OF THE

AMERICAN MATHEMATICAL SOCIETY

Volume 133, Number 9, Pages 2661-2670

S 0002-9939(05)07785-3

Article electronically published on March 21, 2005

\title{
ON EXTENDING EXISTENCE THEORY FROM SCALAR ORDINARY DIFFERENTIAL EQUATIONS TO INFINITE QUASIMONOTONE SYSTEMS OF FUNCTIONAL EQUATIONS
}

\author{
J. ÁNGEL CID \\ (Communicated by Carmen C. Chicone)
}

\begin{abstract}
In this paper we use Tarski's fixed point theorem to extend in a systematic way the existence of extremal solutions from scalar initial value problems to boundary value problems for infinite quasimonotone functional systems of differential equations.
\end{abstract}

\section{INTRODUCTION}

Assuming that we have a result on, roughly speaking, the existence of extremal solutions and comparison principles for scalar initial value problems of the type

$$
\left\{\begin{array}{l}
z^{\prime}(t)=g(t, z(t)) \quad \text { for a.a. } t \in[0, T], \\
z(0)=z_{0},
\end{array}\right.
$$

we can prove, by using Tarski's fixed point theorem, the existence of extremal solutions for infinite functional boundary value problems such as

$$
\left\{\begin{array}{l}
x^{\prime}(t)=f(t, x(t), x) \text { for a.a. } t \in[0, T], \\
x(\theta)=B x(\theta) \text { for all } \theta \in[-r, 0],
\end{array}\right.
$$

under a list of assumptions that we will detail in the next section.

Our motivation is to improve in a unified way the main results in the recent papers [3], 6, 20] and 25].

We prove our main result in section 2. In section 3 we discuss our hypotheses and their relation with the literature. Finally in section 4 we present a particular case covered by our main result.

\section{Preliminaries and main Result}

We say that a partially ordered set (poset) $X$ is a lattice if $\sup \left\{x_{1}, x_{2}\right\}$ and $\inf \left\{x_{1}, x_{2}\right\}$ exist for all $x_{1}, x_{2} \in X$. A lattice $X$ is complete when each nonempty subset $Y \subset X$ has the supremum and the infimum in $X$. In particular, every complete lattice has the maximum and the minimum.

Received by the editors November 19, 2003 and, in revised form, April 22, 2004.

2000 Mathematics Subject Classification. Primary 34A12, 34K10.

Key words and phrases. Functional differential equations, infinite systems, quasimonotone, Tarski's fixed point theorem, existence of extremal solutions.

(C)2005 American Mathematical Society
public domain 28 years from publication Reverts to public domain 28 years from publication 
In a poset $X$ we define for each $a, b \in X$, with $a \leq b$, the interval

$$
[a, b]_{X}:=\{x \in X: a \leq x \leq b\} .
$$

The following is the well-known Tarski's fixed point theorem, [29], which is a fundamental tool in our work.

Theorem 2.1. Every nondecreasing mapping $G: X \rightarrow X$ on a complete lattice $X$ has a minimal, $x_{*}$, and a maximal fixed point, $x^{*}$. Moreover,

$$
x_{*}=\min \{x \in X: G x \leq x\}, \quad x^{*}=\max \{x \in X: x \leq G x\} .
$$

Let $T>0$ and $r>0$ be fixed. We denote by $A C([0, T])$ the set of all functions $x:[0, T] \rightarrow \mathbb{R}$ which are absolutely continuous and by $\mathcal{B}([-r, 0])$ the set of all functions $x:[-r, 0] \rightarrow \mathbb{R}$ which are bounded. Let $M$ be an arbitrary index set and for each $\nu \in M$, let $h_{\nu}:[0, T] \rightarrow \mathbb{R}$ be a Lebesgue-integrable function and define

$$
\begin{gathered}
C_{h_{\nu}}([0, T])=\left\{x:[0, T] \rightarrow \mathbb{R}:|x(s)-x(t)| \leq\left|\int_{s}^{t} h_{\nu}(r) d r\right| \forall s, t \in[0, T]\right\}, \\
\mathcal{S}_{\nu}=\left\{\xi:[-r, T] \rightarrow \mathbb{R}: \xi_{\left.\right|_{[-r, 0]}} \in \mathcal{B}([-r, 0]) \text { and } \xi_{\left.\right|_{[0, T]}} \in C_{h_{\nu}}([0, T])\right\} .
\end{gathered}
$$

We let $\mathcal{S}=\prod_{\nu \in M} \mathcal{S}_{\nu}$. Notice that for every $\nu \in M$ we have that $C_{h_{\nu}}([0, T]) \subset$ $A C([0, T])$. In $C_{h_{\nu}}([0, T])$ and in $\mathcal{S}_{\nu}$ we consider the pointwise partial ordering

$$
\begin{gathered}
x_{1}, x_{2} \in C_{h_{\nu}}([0, T]), \quad x_{1} \leq x_{2} \Longleftrightarrow x_{1}(t) \leq x_{2}(t) \text { for all } t \in[0, T], \\
\xi_{1}, \xi_{2} \in \mathcal{S}_{\nu}, \quad \xi_{1} \leq \xi_{2} \Longleftrightarrow \xi_{1}(t) \leq \xi_{2}(t) \text { for all } t \in[-r, T],
\end{gathered}
$$

and in $\mathcal{S}$ the induced componentwise ordering,

$$
\xi, \eta \in \mathcal{S}, \quad \xi \leq \eta \Longleftrightarrow \xi_{\nu} \leq \eta_{\nu} \text { for all } \nu \in M .
$$

In this paper we are going to study the infinite first-order functional boundary value problem

$$
\left\{\begin{array}{l}
x^{\prime}(t)=f(t, x(t), x) \text { for a.a. } t \in[0, T], \\
x(\theta)=B x(\theta) \text { for all } \theta \in[-r, 0],
\end{array}\right.
$$

where $f:=\left(f_{\nu}\right)_{\nu \in M}:[0, T] \times \mathbb{R}^{M} \times \mathcal{S} \rightarrow \mathbb{R}^{M}$ and $B: \mathcal{S} \rightarrow(\mathcal{B}([-r, 0]))^{M}$.

Definition 2.1. We say that $x:=\left(x_{\nu}\right)_{\nu \in M} \in \mathcal{S}$ is a lower solution of problem (2.1) if for each $\nu \in M$ we have

$$
\left\{\begin{array}{l}
x_{\nu}^{\prime}(t) \leq f_{\nu}(t, x(t), x) \text { for a.a. } t \in[0, T], \\
x_{\nu}(\theta) \leq(B x)_{\nu}(\theta) \text { for all } \theta \in[-r, 0] .
\end{array}\right.
$$

Analogously we say that $x:=\left(x_{\nu}\right)_{\nu \in M} \in \mathcal{S}$ is an upper solution of 2.11) if the above inequalities are reversed. We say that $x:=\left(x_{\nu}\right)_{\nu \in M} \in \mathcal{S}$ is a solution of (2.1) if it is both a lower and an upper solution.

A solution $x^{*} \in A \subset \mathcal{S}$ is a maximal solution in the set $A$ if $x^{*} \geq x$ for any other solution $x \in A$ of (2.1). The minimal solution in $A$ is defined analogously by reversing the inequalities; when both a minimal and a maximal solution in $A$ exist, we call them the extremal solutions in $A$.

For each $\nu \in M$ we denote by $e^{\nu}:=\left(\delta_{\mu}^{\nu}\right)_{\mu \in M}$ the element of $\mathbb{R}^{M}$ with components $\delta_{\mu}^{\nu}=1$, if $\mu=\nu$, and $\delta_{\mu}^{\nu}=0$, if $\mu \neq \nu$. 
Next we present our main result.

Theorem 2.2. Let $f:=\left(f_{\nu}\right)_{\nu \in M}:[0, T] \times \mathbb{R}^{M} \times \mathcal{S} \rightarrow \mathbb{R}^{M}$ and $B: \mathcal{S} \rightarrow$ $(\mathcal{B}([-r, 0]))^{M}$. Assume that there exist $\alpha, \beta \in \mathcal{S}$ with $\alpha \leq \beta$ such that the following hypotheses hold:

(i) For each $\nu \in M$ and each $\xi:=\left(\xi_{\nu}\right)_{\nu \in M} \in[\alpha, \beta]_{\mathcal{S}}$ the scalar initial value problem

$$
\left\{\begin{array}{l}
z^{\prime}(t)=g_{\nu}^{\xi}(t, z(t)) \quad \text { for a.a. } t \in[0, T] \\
z(0)=(B \xi)_{\nu}(0)
\end{array}\right.
$$

where $g_{\nu}^{\xi}:[0, T] \times \mathbb{R} \rightarrow \mathbb{R}$ is defined by

$$
g_{\nu}^{\xi}(t, z):=f_{\nu}\left(t, \xi(t)+\left(z-\xi_{\nu}(t)\right) e^{\nu}, \xi\right) \quad \text { for all }(t, z) \in[0, T] \times \mathbb{R},
$$

has a maximal solution in $A:=\left[\tilde{\alpha_{\nu}}, \tilde{\beta}_{\nu}\right]_{C_{h_{\nu}}([0, T])}, z^{*}$, and a minimal solution in $A, z_{*}$, which moreover satisfy

$$
\begin{aligned}
& z^{*}=\max \left\{z \in A: z^{\prime}(t) \leq g_{\nu}^{\xi}(t, z(t)) \text { a.e. }[0, T], z(0) \leq(B \xi)_{\nu}(0)\right\}, \\
& z_{*}=\min \left\{z \in A: z^{\prime}(t) \geq g_{\nu}^{\xi}(t, z(t)) \text { a.e. }[0, T], z(0) \geq(B \xi)_{\nu}(0)\right\}
\end{aligned}
$$

where $\tilde{\alpha_{\nu}}=\alpha_{\nu_{[0, T]}}$ and $\tilde{\beta}_{\nu}=\beta_{\nu_{[0, T]}}$.

(ii) For each $\nu \in M$, each $\xi \in[\alpha, \beta]_{\mathcal{S}}$ and a.a. $t \in[0, T]$ we have that if $x, y \in \mathbb{R}^{M}$ with $x \leq y$ and $x_{\nu}=y_{\nu}$, then $f_{\nu}(t, x, \xi) \leq f_{\nu}(t, y, \xi)$.

(iii) For each $\nu \in M$, a.a. $t \in[0, T]$ and all $x \in \mathbb{R}^{M}$ the function $f_{\nu}(t, x, \cdot)$ is nondecreasing in $[\alpha, \beta]_{\mathcal{S}}$.

(iv) $B:[\alpha, \beta]_{\mathcal{S}} \rightarrow(\mathcal{B}([-r, 0]))^{M}$ is nondecreasing and moreover

$$
B \alpha(\theta) \geq \alpha(\theta) \quad \text { and } \quad B \beta(\theta) \leq \beta(\theta) \quad \text { for all } \theta \in[-r, 0] .
$$

Then problem (2.1) has a maximal solution, $x^{*}$, and a minimal one, $x_{*}$, in $[\alpha, \beta]_{\mathcal{S}}$. Moreover, we have

$$
\begin{gathered}
x^{*}=\max \left\{x \in[\alpha, \beta]_{\mathcal{S}}: x \text { is a lower solution of }(2.1)\right\}, \\
x_{*}=\min \left\{x \in[\alpha, \beta]_{\mathcal{S}}: x \text { is an upper solution of (2.1) }\right\} .
\end{gathered}
$$

Proof. We shall prove the existence of the maximal solution since the existence of the minimal solution follows from the dual arguments.

Let us consider for each $\nu \in M$ the mapping $G_{\nu}:[\alpha, \beta]_{\mathcal{S}} \rightarrow\left[\alpha_{\nu}, \beta_{\nu}\right]_{\mathcal{S}_{\nu}}$ defined for each $\xi \in[\alpha, \beta]_{\mathcal{S}}$ as follows:

Definition of $G_{\nu} \xi$ on $[-r, 0]$. We define

$$
G_{\nu} \xi(\theta)=(B \xi)_{\nu}(\theta) \text { for all } \theta \in[-r, 0] .
$$

Notice that $\alpha \leq \beta$ and condition (iv) imply $\alpha \leq B \alpha \leq B \xi \leq B \beta \leq \beta$ on $[-r, 0]$ and then $\alpha_{\nu} \leq G_{\nu} \xi \leq \beta_{\nu}$ on $[-r, 0]$.

Definition of $G_{\nu} \xi$ on $[0, T]$. By condition (i) we can define

$\left(G_{\nu} \xi\right)_{\mid[0, T]}:=$ the maximal solution in $\left[\tilde{\alpha_{\nu}}, \tilde{\beta_{\nu}}\right]_{C_{h_{\nu}}([0, T])}$ of the scalar IVP (2.2), and $\left(G_{\nu} \xi\right)_{[0, T]}$ satisfies (2.4).

Therefore by its definition, $G_{\nu} \xi \in\left[\alpha_{\nu}, \beta_{\nu}\right]_{\mathcal{S}_{\nu}}$. Now we consider the mapping $G:=\left(G_{\nu}\right)_{\nu \in M}:[\alpha, \beta]_{\mathcal{S}} \rightarrow[\alpha, \beta]_{\mathcal{S}}$ defined for each $\xi \in[\alpha, \beta]_{\mathcal{S}}$ as $G \xi:=\left(G_{\nu} \xi\right)_{\nu \in M}$. Claim 1. $G:[\alpha, \beta]_{\mathcal{S}} \rightarrow[\alpha, \beta]_{\mathcal{S}}$ is nondecreasing. 
Let $\xi, \eta \in[\alpha, \beta]_{\mathcal{S}}$ be such that $\xi \leq \eta$ and fix $\nu \in M$. By (iv) we have that

$$
G_{\nu} \xi=(B \xi)_{\nu} \leq(B \eta)_{\nu}=G_{\nu} \eta \quad \text { on }[-r, 0] .
$$

On the other hand, $\left(G_{\nu} \xi\right)_{\mid[0, T]} \in\left[\tilde{\alpha_{\nu}}, \tilde{\beta_{\nu}}\right]_{C_{h_{\nu}}([0, T])}$ and by conditions $(i i)$, (iii) and (iv) we deduce

$$
\begin{gathered}
\left(G_{\nu} \xi\right)^{\prime}(t)=g_{\nu}^{\xi}\left(t, G_{\nu} \xi(t)\right) \leq g_{\nu}^{\eta}\left(t, G_{\nu} \xi(t)\right) \text { for a.a. } t \in[0, T] \\
G_{\nu} \xi(0)=(B \xi)_{\nu}(0) \leq(B \eta)_{\nu}(0)
\end{gathered}
$$

which by (2.4) implies that $G_{\nu} \xi \leq G_{\nu} \eta$ on $[0, T]$. Since $\nu \in M$ is arbitrary we conclude that $G \xi \leq G \eta$.

Claim 2. $[\alpha, \beta]_{\mathcal{S}}$ is a complete lattice.

Since $[\alpha, \beta]_{\mathcal{S}}=\prod_{\nu \in M}\left[\alpha_{\nu}, \beta \nu\right]_{\mathcal{S}_{\nu}}$ it suffices to prove that for each $\nu \in M$ the set $\left[\alpha_{\nu}, \beta \nu\right]_{\mathcal{S}_{\nu}}$ is a complete lattice. Let $\emptyset \neq Y \subset\left[\alpha_{\nu}, \beta_{\nu}\right]_{\mathcal{S}_{\nu}}$. We shall prove only the existence of $\sup Y$, because the existence of $\inf Y$ is proved by similar arguments. We define

$$
\xi^{*}(t):=\sup \{\xi(t): \xi \in Y\} \quad \text { for all } t \in[-r, T] .
$$

Since $\alpha_{\nu}(t) \leq \xi(t) \leq \beta_{\nu}(t)$ for all $t \in[-r, T]$ it is clear that $\xi^{*}(t)$ is well defined for all $t \in[-r, T]$ and $\alpha_{\nu} \leq \xi^{*} \leq \beta_{\nu}$ on $[-r, T]$. So $\xi^{*}$ is bounded on $[-r, 0]$. Finally we shall prove that $\xi_{\left.\right|_{[0, T]} ^{*}}^{*} \in C_{h_{\nu}}([0, T])$. Fix $s, t \in[0, T]$ and $\xi \in Y$. Then

$$
\xi(s) \leq|\xi(s)-\xi(t)|+\xi(t) \leq\left|\int_{s}^{t} h_{\nu}(r) d r\right|+\xi^{*}(t) .
$$

Now taking the supremum on the left-hand side we obtain $\xi^{*}(s) \leq\left|\int_{s}^{t} h_{\nu}(r) d r\right|+$ $\xi^{*}(t)$. Interchanging $s$ and $t$ we obtain $\xi^{*}(t) \leq\left|\int_{t}^{s} h_{\nu}(r) d r\right|+\xi^{*}(s)$, and combining these results we have

$$
\left|\xi^{*}(s)-\xi^{*}(t)\right| \leq\left|\int_{s}^{t} h_{\nu}(r) d r\right| .
$$

Therefore $\xi^{*} \in\left[\alpha_{\nu}, \beta_{\nu}\right]_{\mathcal{S}_{\nu}}$ and obviously $\xi^{*}=\sup Y$.

Claims 1 and 2 imply that $G:[\alpha, \beta]_{\mathcal{S}} \rightarrow[\alpha, \beta]_{\mathcal{S}}$ satisfies the conditions of Tarski's fixed point theorem and then $G$ has the maximal fixed point $x^{*}$, which satisfies

$$
x^{*}=\max \left\{x \in[\alpha, \beta]_{\mathcal{S}}: x \leq G x\right\} .
$$

Claim 3. $x^{*}$ is the maximal solution of problem (2.1) in $[\alpha, \beta]_{\mathcal{S}}$ and moreover satisfies (2.6).

Indeed, by the definition of $G$ it follows that $x^{*}$ is a solution of (2.1). Suppose now that $x:=\left(x_{\nu}\right)_{\nu \in M} \in[\alpha, \beta]_{\mathcal{S}}$ is a lower solution for (2.1), i.e., for each $\nu \in M$ we have that

$$
x_{\nu}^{\prime}(t) \leq f_{\nu}(t, x(t), x) \text { a.e. in }[0, T], x_{\nu}(\theta) \leq(B x)_{\nu}(\theta) \text { for all } \theta \in[-r, 0] .
$$

Then by the definition of $G$, (2.4) and (2.9) we have that $x \leq G x$, and thus by (2.8) we deduce that $x \leq x^{*}$. Moreover, since $x^{*}$ is solution of (2.1), and in particular $x^{*}$ is a lower solution of (2.1), we obtain (2.6). 


\section{REMARKS ON THE HYPOTHESES}

1. Condition (i) in Theorem 2.2 looks difficult to verify; however, there are in the literature a lot of sufficient conditions which imply the existence of extremal solutions satisfying the comparison properties (2.4) and (2.5) for scalar initial value problems of the type

$$
\left\{\begin{array}{l}
z^{\prime}(t)=g(t, z(t)) \quad \text { for a.a. } t \in[0, T] \\
z(0)=z_{0}
\end{array}\right.
$$

where $g:[0, T] \times \mathbb{R} \rightarrow \mathbb{R}$ and $z_{0} \in \mathbb{R}$.

Let us start by mentioning Carathéodory, 4, who proved that whenever $g$ : $[0, T] \times \mathbb{R} \rightarrow \mathbb{R}$ satisfies

$(C 1)$ for all $z \in \mathbb{R}, g(\cdot, z)$ is measurable on $[0, T]$,

$(C 2)$ for a.a. $t \in[0, T], g(t, \cdot)$ is continuous on $\mathbb{R}$,

(C3) there exists $h \in L^{1}(0, T)$ such that for a.a. $t \in[0, T]$ and all $z \in \mathbb{R}$,

$$
|g(t, z)| \leq h(t),
$$

then problem (3.10) has at least one absolutely continuous solution (even in the finite-dimensional case). By using Peano's and Perron's approach, [23, 24], Goodman improved in [10] the Carathéodory result in the scalar case, proving that the function $z^{*}$ defined for all $t \in[0, T]$ as

$$
z^{*}(t)=\sup \left\{z(t): z \in A C([0, T]), z^{\prime}(s) \leq g(s, z(s)) \text { a.e., } z(0) \leq z_{0}\right\},
$$

is a solution of (3.10). Clearly, this result includes the comparison theorem for differential inequalities and characterizes $z^{*}$ as the maximal solution. An analogous theorem holds of course for the minimal solution $z_{*}$. Moreover $z_{*}$ and $z^{*}$ are the extremal solutions in the functional interval $[\tilde{\alpha}, \tilde{\beta}]_{C_{h}([0, T])}$ where

$$
\tilde{\alpha}(t):=z_{0}-\int_{0}^{t} h(s) d s \quad \text { and } \quad \tilde{\beta}(t):=z_{0}+\int_{0}^{t} h(s) d s \quad \text { for all } t \in[0, T],
$$

and $h$ is the function given in condition $(C 3)$.

Goodman's result has been extensively generalized in recent years [2, 8, 13. 18 27. One essential step is due to Hassan and Rzymowski, [13, who proved the existence of the extremal solutions satisfying the corresponding comparison principles under the assumptions $(C 1),(C 3)$, and

$(H R)$ for a.a. $t \in[0, T]$ and all $z \in \mathbb{R}$, we have

$$
\limsup _{y \rightarrow z^{-}} g(t, y) \leq g(t, z) \leq \liminf _{y \rightarrow z^{+}} g(t, z) .
$$

Using a revision of Hassan and Rzymowski's arguments Pouso showed in [18] that $(H R)$ may fail along a finite number of curves in the $(t, x)$-plane and in a recent paper Cid and Pouso, [8, theorem 3.1], proved that $(H R)$ may fail even along countably many curves, but regrettably in this last reference the condition

$(C P)$ for all $z \in A C([0, T])$, the composition $f(\cdot, z(\cdot))$ is measurable,

which is stronger than $(C 1)$, is needed. Condition $(C P)$ is a type of "superposition-measurability", and it arises in a natural way in the study of discontinuous differential equations (see [1, 2, 3]). It is well known that conditions $(C 1)$ and $(C 2)$ 
imply $(C P)$. However conditions $(C 1)$ and $(H R)$ do not imply $(C P)$, as is shown with a counterexample in [1].

For positive $g$ 's Carl and Heikkilä improved Hassan and Rzymowski's result in the monograph [5] and Cid and Pouso gave in [7 an alternative result which, roughly speaking, interchanges the roles of $t$ and $x$ in the assumptions.

This incomplete and brief overview shows nevertheless that there is a great number of results that we can use to check condition $(i)$. In this way, Theorem 2.2 immediately extends to functional infinite systems any existence and comparison result for scalar initial value problems.

2. Condition (ii) in Theorem 2.2 is generally known as "quasimonotonicity", name coined by Walter, 31, but in some contexts the term cooperative also is used. The first author who used this property seems to be Müller, 22], and since then, quasimonotonicity has been the key for extending several results about differential equations and inequalities from the scalar case to higher dimensions 3, 9, 17, 21, 28, 31, 32]. Quasimonotonicity is also important for extremal fixed points of discontinuous maps [11, 16, 26, 30]. (For a recent survey on quasimonotonicity, see [15].)

3. In our paper we consider a differential equation with functional dependence. This dependence includes some of the most important kinds of functional differential equations: delay differential equations and the equations with maxima (see [12]). On the other hand, the functional boundary condition considered is the same as that in [20]. It includes the ordinary initial condition $x(0)=x_{0}$ as well as several types of periodic conditions, which have more interest, such as the ordinary periodic condition $x(0)=x(T)$ and the functional periodic condition $x(\theta)=x(\theta+T)$ for all $\theta \in[-r, 0]$.

We can also consider for each $\nu \in M$ the integral boundary conditions

$$
x_{\nu}(0)=\int_{0}^{T} x_{\nu}(s) d s \quad \text { or } \quad \gamma_{\nu}=\int_{0}^{T} x_{\nu}(s) d s,
$$

where $\gamma_{\nu}$ is a real constant (this last condition was suggested in [19]).

It is remarkable that we don't need any assumption about the compactness of operator $B$ (compare with [5, section 2.4]). Regrettably such a boundary condition

as $x_{\nu}(0)=\int_{-r}^{\frac{T}{2}} x_{\nu}(s) d s$ for each $\nu \in M$ is not included in our theorem because the operator

$$
(B \xi)_{\nu}(\theta):=\int_{-r}^{\frac{T}{2}} \xi_{\nu}(s) d s \text { for all } \theta \in[-r, 0]
$$

is not defined for all $\xi \in \mathcal{S}$ since $\xi_{\nu}$ need not be Lebesgue-measurable on $[-r, 0]$. Obviously this operator can be defined in the smaller set $\hat{\mathcal{S}}=\prod_{\nu \in M} \hat{\mathcal{S}_{\nu}} \subset \mathcal{S}$ where

$$
\hat{\mathcal{S}_{\nu}}=\left\{\xi \in \mathcal{S}_{\nu}: \xi_{\left.\right|_{[-r, 0]}} \text { is Lebesgue-measurable }\right\},
$$

but Theorem 2.2 is false if we simply replace $\mathcal{S}$ by $\hat{\mathcal{S}}$ (a counterexample is shown in [6]).

\section{A particular case}

In this section we extend, by using Theorem 2.2, the scalar existence theorem [20. theorem 2.4] to an existence result for problem (2.1) and in this way we generalize [3, theorem 1.1], [20, theorem 3.3] (see also [6]) and [25, theorem 2]). 
Theorem 4.1. Let $f:=\left(f_{\nu}\right)_{\nu \in M}:[0, T] \times \mathbb{R}^{M} \times \mathcal{S} \rightarrow \mathbb{R}^{M}$ and $B: \mathcal{S} \rightarrow$ $(\mathcal{B}([-r, 0]))^{M}$. Let $\alpha:=\left(\alpha_{\nu}\right)_{\nu \in M}, \beta:=\left(\beta_{\nu}\right)_{\nu \in M} \in \mathcal{S}$ with $\alpha \leq \beta$ and assume hypotheses (ii), (iii), (iv) and

$\left(i^{\prime}\right)$ For each $\nu \in M$ and each $\xi:=\left(\xi_{\mu}\right)_{\mu \in M} \in[\alpha, \beta]_{\mathcal{S}}$ we have:

(a) For all $z \in \mathbb{R}$ the function $t \rightarrow f_{\nu}\left(t, \xi(t)+\left(z-\xi_{\nu}(t)\right) e^{\nu}\right.$, $\left.\xi\right)$ is measurable on $[0, T]$.

(b) For a.a. $t \in[0, T]$ and all $x:=\left(x_{\mu}\right)_{\mu \in M} \in \mathbb{R}^{M}$ we have

$\limsup _{y \rightarrow x_{\nu}^{-}} f_{\nu}\left(t, x+\left(y-x_{\nu}\right) e^{\nu}, \xi\right) \leq f_{\nu}(t, x, \xi) \leq \liminf _{y \rightarrow x_{\nu}^{+}} f_{\nu}\left(t, x+\left(y-x_{\nu}\right) e^{\nu}, \xi\right)$.

(c) For a.a. $t \in[0, T]$ we have

$$
\left|f_{\nu}(t, x, \xi)\right| \leq h_{\nu}(t) \quad \text { for all } \alpha(t) \leq x \leq \beta(t) .
$$

(d) For a.a. $t \in[0, T]$ we have

$$
\alpha_{\nu}^{\prime}(t) \leq f_{\nu}(t, \alpha(t), \alpha) \quad \text { and } \quad \beta_{\nu}^{\prime}(t) \geq f_{\nu}(t, \beta(t), \beta) .
$$

Then problem (2.1) has a maximal solution, $x^{*}$, and a minimal one, $x_{*}$, in $[\alpha, \beta]_{\mathcal{S}}$. Moreover, we have

$$
\begin{gathered}
x^{*}=\max \left\{x \in[\alpha, \beta]_{\mathcal{S}}: x \text { is a lower solution of }(2.1)\right\}, \\
x_{*}=\min \left\{x \in[\alpha, \beta]_{\mathcal{S}}: x \text { is an upper solution of (2.1) }\right\} .
\end{gathered}
$$

Proof. We only have to prove that condition $(i)$ of Theorem 2.2 follows from our assumptions. Fix $\nu \in M$ and $\xi \in[\alpha, \beta]_{\mathcal{S}}$.

By conditions $\left(i^{\prime}\right)-(d),(i i),($ iii $)$ and $(i v)$, we obtain

$$
\begin{gathered}
\alpha_{\nu}^{\prime}(t) \leq f_{\nu}(t, \alpha(t), \xi) \leq f_{\nu}\left(t, \xi(t)+\left(\alpha_{\nu}(t)-\xi_{\nu}(t)\right) e^{\nu}, \xi\right)=g_{\nu}^{\xi}\left(t, \alpha_{\nu}(t)\right) \text { a.e. on }[0, T], \\
\alpha_{\nu}(0) \leq(B \alpha)_{\nu}(0) \leq(B \xi)_{\nu}(0),
\end{gathered}
$$

and the reversing inequalities are deduced for $\beta_{\nu}$. Then ${\tilde{\alpha_{\nu}}}_{\nu}:=\alpha_{\left.\nu\right|_{[0, T]}}$ and $\tilde{\beta}_{\nu}:=$ $\beta_{\nu_{[0, T]}}$ are lower and upper solutions, respectively, for the initial value problem (2.2) (see [20, definition 2.3] where the concept of lower and upper solutions for problem (2.2) is defined). Moreover condition $\left(i^{\prime}\right)$ implies

1. For all $z \in \mathbb{R}$ the function $g_{\nu}^{\xi}(\cdot, z)$ is measurable on $[0, T]$.

2. For a.a. $t \in[0, T]$ and all $z \in \mathbb{R}$ we have

$$
\begin{aligned}
\limsup _{y \rightarrow z^{-}} g_{\nu}^{\xi}(t, y) & =\limsup _{y \rightarrow z^{-}} f_{\nu}\left(t, \xi(t)+\left(y-\xi_{\nu}(t)\right) e^{\nu}, \xi\right) \\
& \leq f_{\nu}\left(t, \xi(t)+\left(z-\xi_{\nu}(t)\right) e^{\nu}, \xi\right)=g_{\nu}^{\xi}(t, z) \\
& \leq \liminf _{y \rightarrow z^{+}} f_{\nu}\left(t, \xi(t)+\left(y-\xi_{\nu}(t)\right) e^{\nu}, \xi\right)=\liminf _{y \rightarrow z^{+}} g_{\nu}^{\xi}(t, y) .
\end{aligned}
$$

3. For a.a. $t \in[0, T]$ we have

$$
\left|g_{\nu}^{\xi}(t, z)\right| \leq h_{\nu}(t) \quad \text { for all } \tilde{\alpha}(t) \leq z \leq \tilde{\beta}(t) .
$$

Then adapting [13, theorem 3.1] to the case of lower and upper solutions in the same way as in [20, theorem 2.4], we obtain the existence of the extremal solutions for the initial value problem (2.2) satisfying (2.4) and (2.5).

Remarks. 1. Biles and Schechter studied in [3, theorem 1.1] the quasimonotone infinite system (2.1) without functional dependence and considering only the initial condition $x(0)=0$. Their method for proving the existence of solutions consists in taking the supremum of subsolutions and showing that this supremum is a solution. 
To accomplish this they use the measure-theoretic techniques used for the onedimensional case in [27].

In [25, theorem 2] Pikuta and Rzymowski extended to functional differential equations the result of Biles and Schechter (only for the finite-dimensional case). Their proof relies directly on Hassan and Rzymowski's result [13, theorem 3.1] for scalar initial value problems.

Liz and Pouso considered in [20, theorem 3.3] (see also [6]) the problem (2.1) (only for the scalar case $M=1$ ) introducing the general boundary functional condition $\xi(\theta)=B \xi(\theta)$ for all $\theta \in[-r, 0]$ and considering lower and upper solutions. Their proof is based on a fixed point theorem, which is given in [14].

We point out that although these three results were proved by different methods and in different contexts, our Theorem 4.1 improves all of them at one stroke with a unified technique.

2. For the scalar case $(M=1)$ condition $(i)^{\prime}-(a)$ is simply

(I) For all $z \in \mathbb{R}$ the function $f(\cdot, z, \xi)$ is measurable on $[0, T]$.

We can wonder whether Theorem4.1 is still true when weakening condition $(i)^{\prime}-(a)$ to the multidimensional analogue of $(I)$, that is,

(II) For all $z \in \mathbb{R}^{M}$ the function $f_{\nu}(\cdot, z, \xi)$ is measurable on $[0, T]$.

The answer in general is negative, even for $M=2$, as we show in the following counterexample.

Counterexample: Let $S$ be any nonmeasurable set such that $S \subset[0,1]$ and define $g:[0,1] \times \mathbb{R} \rightarrow \mathbb{R}$ by

$$
g(t, z)= \begin{cases}1, & t \in[0,1], z>t \\ 1, & t \in S, z=t \\ 0, & \text { otherwise. }\end{cases}
$$

(This function was used by Biles in [1].) Consider now the system

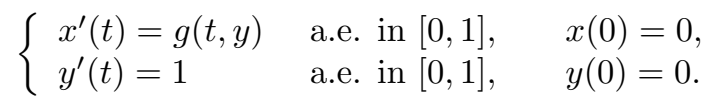

This system satisfies the assumptions of Theorem 4.1 replacing condition $\left(i^{\prime}\right)-(a)$ by $(I I)$. Nevertheless it is easy to see that it has no solution.

\section{ACKNOWLEDGEMENT}

The author wants to thank Prof. Rodrigo L. Pouso for his valuable comments and fruitful discussions about this paper.

\section{REFERENCES}

[1] D.C. Biles, Existence of solutions for discontinuous differential equations, Differential Integral Equations 8 (1995), 1525-1532. MR1329854 (96d:34003)

[2] D.C. Biles \& P.A. Binding, On Carathéodory's conditions for the initial value problem, Proc. Amer. Math. Soc. 125, 5 (1997), 1371-1376. MF1403114 (97g:34008)

[3] D.C. Biles \& E. Schechter, Solvability of a finite or infinite system of discontinuous quasimonotone differential equations, Proc. Amer. Math. Soc. 128, 5 (2000), 3349-3360. MR:1707137|(2001b:34015)|

[4] C. Carathéodory, Vorlesungen über reelle Funktionen, Chelsea Publishing Company, New York (1968), Third Edition. Original edition: Leipzig (1918). MR0225940 (37:1530) 
[5] S. Carl \& S. Heikkilä, "Nonlinear Differential Equations in Ordered Spaces", Chapman \& Hall/CRC, 2000. MR1773963 (2001e:34112)

[6] J.A. Cid, E. Liz \& R. L. Pouso, Corrigendum to "Existence theory for first order discontinuous functional differential equations", by E. Liz and R. L. Pouso, Proc. Amer. Math. Soc. 132, 10 (2004), 3135-3136. MR2063137 (2005c:34005)

[7] J.A. Cid \& R.L. Pouso, On first order ordinary differential equations with non-negative right-hand sides, Nonlinear Anal., 52 (2003), 1961-1977. MF1954592 (2003k:34008)

[8] J.A. Cid \& R.L. Pouso, Ordinary differential equations and systems with time-dependent discontinuity sets, Proc. Roy. Soc. Edinburgh Sect. A 134, 4 (2004), 617-637. MR2079796

[9] A. Chaljub-Simon \& P. Volkmann, Un théorème d'existence et de comparaison pour des équations différentielles dans les espaces de fonctions bornées, C. R. Acad. Sci. Paris Sér. I Math., 311 (1990), no. 9, 515-517. MF 1078113 (91h:34099)

[10] G.S. Goodman, Subfunctions and the initial-value problem for differential equations satisfying Carathéodory's hypotheses, J. Differential Equations 7 (1970), 232-242. MR0255880 $(41: 540)$

[11] J. Guillerme, Intermediate value theorems and fixed point theorems for semi-continuous functions in product spaces, Proc. Amer. Math. Soc. 123, (1995), 2119-2122. MR 1246525 (95i:54053)

[12] J. K. Hale \& S. M. Verduyn Lunel, Introduction to functional differential equations, Springer-Verlag, New York, 1993. MR 1243878 (94m:34169)

[13] E.R. Hassan \& W. Rzymowski, Extremal solutions of a discontinuous scalar differential equation, Nonlinear Anal. 37 (1999), 997-1017. MF,1689280 (2000d:34015)

[14] S. Heikkilä \& V. Lakshmikantham, Monotone iterative techniques for discontinuous nonlinear differential equations, Marcel Dekker, New York, 1994. MR1280028 (95d:34002)

[15] G. Herzog, Quasimonotonicity, Nonlinear Anal. 47, (2001), 2213-2224. Proceedings of the Third World Congress of Nonlinear Analysts, Part 4 (Catania, 2000). MR1971631 $(2004 \mathrm{c}: 47104)$

[16] S. Hu, Fixed points for discontinuous quasi-monotone maps in $\mathbb{R}^{n}$, Proc. Amer. Math. Soc. 104, (1988), 1111-1114. MR.0937846 (89k:47093)

[17] E. Kamke, Zur theorie der systeme gewöhnlicher differentialgleichungen II, Acta Math., 58 (1932), 57-85.

[18] R. López Pouso, On the Cauchy problem for first order discontinuous ordinary differential equations, J. Math. Anal. Appl. 264, 1 (2001), 230-252. MR.1868338(2002i:34004)

[19] J. W. Lee \& D. O'Regan, Existence results for differential delay equations, I, J. Differential Equations 102 (1993), 342-359. MR,1216733(94c:34096)

[20] E. Liz \& R. L. Pouso, Existence theory for first order discontinuous functional differential equations, Proc. Amer. Math. Soc. 130, 11 (2002), 3301-3311. MR1913010 (2004d:34012)

[21] W. Mlak \& C. Olech, Integration of infinite systems of differential inequalities, Ann. Polon. Math, 13 (1963), 105-112. MR0147735 (26:5249)

[22] M. Müller, Über das fundamentaltheorem in der theorie der gewöhnlichen differentialgleichungen, Math. Z., 26 (1926), 619-645.

[23] G. Peano, Sull'integrabilità delle equazione differenziali di primo ordine, Atti. Acad. Torino $21(1885 / 6), 667-685$.

[24] O. Perron, Ein neuer existzbeweis für die integrale der differentialgleichung $y^{\prime}=f(x, y)$, Math. Ann. 76 (1914/5), 471-484.

[25] P. Pikuta \& W. Rzymowski, A discontinuous functional differential equation, J. Math. Anal. Appl. 277 (2003), 122-129. MR.1954465(2004a:34109)

[26] S. Schmidt, Fixed points for discontinuous quasi-monotone maps in sequence spaces, Proc. Amer. Math. Soc. 115 (1992), no. 2, 361-363. MF1081098 (93c:47072)

[27] W. Rzymowski \& D. Walachowski, One-dimensional differential equations under weak assumptions, J. Math. Anal. Appl., 198, (1996), 657-670. MR,1377818 (97c:34009)

[28] J. Szarski, Sur les systèmes majorants d'équations différentielles ordinaires, Ann. Soc. Polon. Math. 23 (1950), 206-223. MRMR0040507 (12:705b)

[29] A. Tarski, A lattice-theoretical fixed point theorem and its applications, Pacific J. Math. 5, (1955), 285-309. MR0074376 (17:574d)

[30] R. Uhl, Smallest and greatest fixed points of quasimonotone increasing mappings, Math. Nachr., 248-249 (2003), 204-210. MR.1950728 (2003j:47079) 
[31] W. Walter, Differential and integral inequalities, Springer-Verlag, New York, 1970. MR0271508 (42:6391)

[32] T. Ważewski, Systèmes des équations et des inégalités différentielles ordinaires aux deuxiémes membres monotones et leurs applications, Ann. Soc. Polon. Math. 23 (1950), 112-166. MR0040506(12:705a)

Departamento de Análise Matemática, Facultade de Matemáticas, Campus Sur, Universidade de Santiago de Compostela, 15782 Santiago de Compostela, Spain

E-mail address: angelcid@usc.es

Current address: Departamento de Matemáticas, Escuela Universitaria Politécnica de Linares, Universidad de Jaén, c/Alfonso X el Sabio, no. 28, 23700, Linares (Jaén), Spain

E-mail address: angelcid@ujaen.es 